\title{
Very rare, exclusive, hadronic decays in QCD factorization
}

\author{
Matthias König ${ }^{1, \star}$ \\ ${ }^{1}$ Mainz Institute for Theoretical Physics, JGU Mainz
}

\begin{abstract}
We study exclusive hadronic decays of the electroweak bosons $Z, W$ and $h$ in the framework of QCD factorization. We show that the theory uncertainties in these channels are remarkably small compared to past applications of the QCD factorization framework. While the branching ratios are small, many of the modes are accessible at future colliders. The Higgs decays exhibit interesting dependences on the couplings due to the interferences of different diagram topologies, making the $h \rightarrow V \gamma$ decays possible probes of the quark Yukawa couplings and the $h \rightarrow V Z$ decays probes of the coupling between the Higgs boson, a photon and a $Z$-boson.
\end{abstract}

Talk based on work in collaboration with Stefan Alte, Yuval Grossman and Matthias Neubert [1-4].

\section{Introduction}

The Standard Model (SM) of particle physics is in remarkable agreement with observation and the discovery of the Higgs boson in 2012 [5, 6] is arguably the biggest triumph of particle physics in recent times. Still many questions remain unanswered and thus the SM has to be extended. When doing so, the Higgs sector is usually modified, making it a crucial experimental task to measure the parameters in this sector as accurately as possible. The exclusive hadronic decays of the form $h \rightarrow M \gamma, h \rightarrow M Z$ and $h \rightarrow M W$ can be used to probe several different couplings of the Higgs boson to the SM fermions and gauge bosons $[2,7,8]$.

Even within the SM, particle physics still faces the challenge of obtaining rigorous control over non-perturbative effects in QCD at low energy scales. The framework of QCD factorization is a wellestablished approach to hard exclusive processes with indiviual final state hadrons [9-13]. Within this framework, amplitudes are given as convolutions of hard-scattering functions with non-local hadronic matrix elements, which encode the non-perturbative physics at the low scale. The amplitudes will formally be given as expansions in the ratio of the two scales. Past applications struggled with the fact that this factorization scale was not high enough for power corrections to be neglected and that it was difficult to disentangle these uncertainties from those in the poorly-known hadronic input parameters. In our case, the scale is set by the decaying heavy boson and power corrections are well under control. The decays $Z \rightarrow M \gamma$ and $W \rightarrow M \gamma$ can be used to test that factorization approach in a theoretically clean environment $[1,3]$.

\footnotetext{
^e-mail: m.koenig@uni-mainz.de
} 

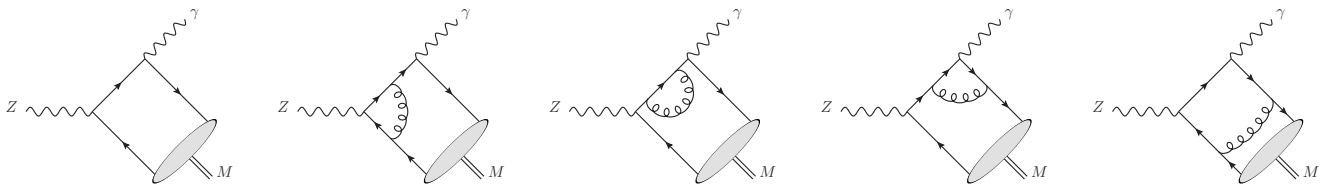

Figure 1. A subset of the diagrams contributing to $Z \rightarrow M \gamma$ at $O\left(\alpha_{s}\right)$. Additional mirrored diagrams exist.

\section{QCD factorization}

In the framework of QCD factorization, the amplitude for a hard exclusive process can be written as a convolution of a hard scattering function with a hadronic function, where the result will formally be an expansion of the scale separation parameter $\lambda \sim \Lambda_{\mathrm{QCD}} / \mu_{\text {hard }}$. While the original derivation of the factorization formula dates back to the early 1980s, it can be rephrased in the language of SoftCollinear Effective Theory (SCET) [14-17]. The factorization formula for the decays of $h / Z \rightarrow M V$ the final state meson $M$ and the gauge boson $V$ can be written as:

$$
i \mathcal{A}=\sum_{q} \int d x T_{H}^{q}(x, \mu) \phi_{M}^{q}(x, \mu)+O(\lambda),
$$

where $T_{H}^{q}(x, \mu)$ is the hard-scattering kernel, $\phi_{M}^{q}(x, \mu)$ is the light-cone distribution amplitude (LCDA) and the sum runs over the active quark flavors. Power corrections of higher orders in $\lambda$ are tiny in our case due to the high scale $\mu_{\text {hard }}$.

The LCDAs are expanded in the set of Gegenbauer polynomials

$$
\phi_{M}^{q}(x, \mu)=6 x \bar{x}\left[1+\sum_{n=2,4, \ldots} a_{n}^{M, q}(\mu) C_{n}^{(3 / 2)}(2 x-1)\right],
$$

where $a_{n}^{M, q}(\mu)$ are the scale-dependent Gegenbauer moments [9, 13, 18-21]. By evolving these parameters from the hadronic scale to the high scale using the renormalization group, large logarithms of the form $\alpha_{s} \log \lambda$ are resummed to all orders. It is noteworthy that the evolution to an arbitrarily high scale lets all moments $a_{n}^{M, q}$ vanish, decreasing the sensitivity of our prediction to these poorly-known hadronic parameters.

\section{Radiative hadronic decays of Z-bosons}

We can now write down the amplitude for the decays $Z \rightarrow M \gamma$. We will neglect the modes $Z \rightarrow \eta^{(\prime)} \gamma$ here since the flavor-singlet components lead to non-trivial matching conditions that are too involved to explain here [3]. The amplitude of a $Z$-boson decaying into a meson $M$ with momentum $k$ and a photon with momentum $q$ can be written as:

$$
i \mathcal{A}= \pm \frac{e g}{2 c_{W}}\left[i \epsilon_{\mu \nu \alpha \beta} \frac{k^{\mu} q^{v} \varepsilon_{Z}^{\alpha} \varepsilon_{\gamma}^{* \beta}}{k \cdot q} F_{1}^{M}-\left(\epsilon_{Z}^{\perp} \cdot \epsilon_{\gamma}^{\perp *}\right) F_{2}^{M}\right] .
$$

We can express the form factors as sums over the Gegenbauer moments and the hard scattering coefficients:

$$
F_{1}^{M}=Q_{M} \sum_{n=0}^{\infty} C_{2 n}^{(+)}\left(m_{Z}, \mu\right) a_{2 n}^{M}(\mu), \quad F_{2}^{M}=-Q_{M}^{\prime} \sum_{n=0}^{\infty} C_{2 n+1}^{(-)}\left(m_{Z}, \mu\right) a_{2 n+1}^{M}(\mu),
$$


Table 1. Predicted branching fractions for various $Z \rightarrow M \gamma$ decays, including error estimates due to scale dependence (subscript " $\mu$ ") and the uncertainties in the meson decay constants (" $f$ "), and the shape parameters of the LCDA (“ $\phi ”)$.

\begin{tabular}{|c|c|}
\hline Decay mode & Branching ratio \\
\hline$Z^{0} \rightarrow \pi^{0} \gamma$ & $\left(9.80_{-0.14}^{+0.09} \mu \pm 0.03_{f} \pm 1.02_{\phi}\right) \cdot 10^{-12}$ \\
$Z^{0} \rightarrow \eta \gamma$ & $\left(2.36_{-0.04}^{+0.02} \pm 1.19_{f} \pm 0.04_{\phi}\right) \cdot 10^{-10}$ \\
$Z^{0} \rightarrow \eta^{\prime} \gamma$ & $\left(6.68_{-0.11}^{+0.08} \pm 0.49_{f} \pm 0.12_{\phi}\right) \cdot 10^{-9}$ \\
$Z^{0} \rightarrow \rho^{0} \gamma$ & $\left(4.19_{-0.06}^{+0.04} \pm 0.16_{f} \pm 0.44_{\phi}\right) \cdot 10^{-9}$ \\
$Z^{0} \rightarrow \omega \gamma$ & $\left(2.89_{-0.05}^{+0.03} \pm 0.15_{f} \pm 0.38_{\phi}\right) \cdot 10^{-8}$ \\
$Z^{0} \rightarrow \phi \gamma$ & $\left(8.63_{-0.13}^{+0.08} \pm 0.41_{f} \pm 0.92_{\phi}\right) \cdot 10^{-9}$ \\
$Z^{0} \rightarrow J / \psi \gamma$ & $\left(8.02_{-0.15}^{+0.14} \mu \pm .20_{f}^{+0.39}\right) \cdot 10^{-8}$ \\
$Z^{0} \rightarrow \Upsilon(1 S) \gamma$ & $\left.\left(5.39_{-0.10}^{+0.10} \mu \pm 0.08_{f}^{+0.11}\right) \cdot 10_{-0.08}^{-8}\right) \cdot 10^{-8}$ \\
$Z^{0} \rightarrow \Upsilon(4 S) \gamma$ & $\left(1.22_{-0.02}^{+0.02} \pm 0.13_{f}^{+0.02} \phi\right) \cdot 10^{-8}$ \\
$Z^{0} \rightarrow \Upsilon(n S) \gamma$ & $\left(9.96_{-0.19}^{+0.18} \mu \pm 0.09_{f}^{+0.20}\right) \cdot 10^{-8}$ \\
\hline
\end{tabular}

where $Q^{(\prime)}$ are combinations of the meson decay constants and the quark couplings to the $Z$-boson and the photon. Evaluating diagrams like the ones shown in figure 1, we find the hard scattering coefficients:

$$
\begin{aligned}
C_{n}^{( \pm)}\left(m_{V}, \mu\right)=1 & +\frac{C_{F} \alpha_{s}(\mu)}{4 \pi} c_{n}^{( \pm)}\left(\frac{m_{V}}{\mu}\right)+O\left(\alpha_{s}^{2}\right) \\
\text { with } \quad c_{n}^{( \pm)}\left(\frac{m_{V}}{\mu}\right)=[ & \left.\frac{2}{(n+1)(n+2)}-4 H_{n+1}+3\right]\left(\log \frac{m_{V}^{2}}{\mu^{2}}-i \pi\right) \\
& +4 H_{n+1}^{2}-\frac{4\left(H_{n+1}-1\right) \pm 1}{(n+1)(n+2)}+\frac{2}{(n+1)^{2}(n+2)^{2}}-9 .
\end{aligned}
$$

This is the leading result in our expansion in the scale separation parameter $\lambda$, with subleading corrections starting at $O\left(m_{V}^{2} / m_{Z}^{2}\right)$, that we can safely neglect. We present our phenomenological results in table 1.

Similar results are obtained for the $W \rightarrow M \gamma$ decays. We omit a discussion of these for briefness and refer the reader to ref. [1] for details.

\section{Exclusive hadronic decays of the Higgs boson}

In order to investigate the hadronic decays of the Higgs boson and understand to what extend new physics can manifest itself in them, we work in an effective Lagrangian framework, where we allow a rescaling of the relevant parameters in the SM as well as including higher-dimensional operators:

$$
\begin{aligned}
\mathcal{L}_{\mathrm{eff}}^{\mathrm{Higgs}}= & \kappa_{W} \frac{2 m_{W}^{2}}{v} h W_{\mu}^{+} W^{-\mu}+\kappa_{Z} \frac{m_{Z}^{2}}{v} h Z_{\mu} Z^{\mu}-\sum_{\hat{k}_{\kappa_{\gamma Z}}} \frac{m_{f}}{v} h \bar{f}\left(\kappa_{f}+i \tilde{\kappa}_{f} \gamma_{5}\right) f \\
& +\frac{\alpha}{4 \pi v}\left(\kappa_{\gamma \gamma} h F_{\mu \nu} F^{\mu \nu}-\tilde{\kappa}_{\gamma \gamma} h F_{\mu \nu} \tilde{F}^{\mu \nu}+\frac{2 \tilde{\kappa}_{\gamma Z}}{s_{W} c_{W}} h F_{\mu \nu} \tilde{Z}^{\mu \nu}\right) .
\end{aligned}
$$



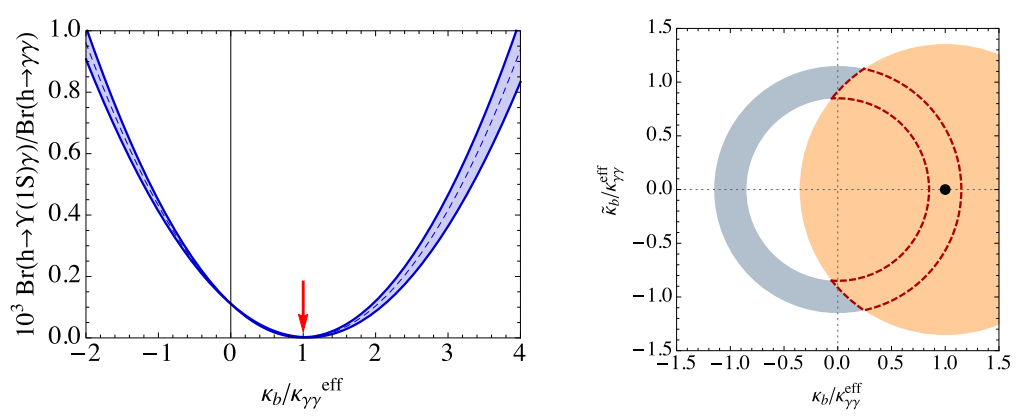

Figure 2. Left diagram: The dependence of $\operatorname{Br}(h \rightarrow \Upsilon(1 S) \gamma)$ on the rescaling of the $b$-quark Yukawa coupling under the assumption that all other parameters take their SM values. Right diagram: An upper bound on $\operatorname{Br}(h \rightarrow$ $\Upsilon(1 S) \gamma)$ constrains the $b$-quark Yukawa to the orange region in the $\kappa_{b}-\tilde{\kappa}_{b}$ plane, whereas the measurement of $\operatorname{Br}(h \rightarrow \bar{b} b)$ constrains only the absolute value $\kappa_{b}^{2}+\tilde{\kappa}_{b}^{2}$, giving the blue band. The overlap region yields the allowed range for the $b$-quark Yukawa coupling in the CP-plane.

In the SM, the parameters $\kappa_{W}, \kappa_{Z}$ and $\kappa_{f}$ are equal to 1 whereas all other $\kappa^{\prime}$ 's vanish.

In the following, we only briefly state the main results of our analysis and direct the interested reader towards refs. [2, 4].

\subsection{Radiative hadronic decays of the Higgs}

The case of $h \rightarrow V \gamma$ is similar to the Z-boson decays, with the difference of an important additional contribution, where the Higgs decays into a pair of photons or into a photon and a $Z$-boson. Here, either the off-shell photon or Z-boson converts to the final state meson. The $h \gamma \gamma$ - and $h \gamma Z$-couplings are generated at 1-loop in the SM or can occur at tree-level in our effective Lagrangian [22]. Generally, these "indirect" contributions are dominant over the ones that directly involve the Yukawa couplings of the valence quarks of the vector meson $V$. When one wants to use these decays to probe said Yukawa couplings, it is useful to normalize the branching ratios to the branching ratio of $h \rightarrow \gamma \gamma$, since all NP effects in the indirect contribution will affect $h \rightarrow \gamma \gamma$ in the same way (up to small corrections due to the off-shellness of the photon and the Z-boson diagram). Expanding in small parameters, we find:

$$
\frac{\operatorname{Br}(h \rightarrow V \gamma)}{\operatorname{Br}(h \rightarrow \gamma \gamma)}=\frac{\Gamma(h \rightarrow V \gamma)}{\Gamma(h \rightarrow \gamma \gamma)}=\frac{8 \pi \alpha_{\mathrm{EM}}^{2}\left(m_{V}\right)}{\alpha_{\mathrm{EM}}(0)} \frac{Q_{V}^{2} f_{V}^{2}}{m_{V}^{2}}\left(1-\frac{m_{V}^{2}}{m_{h}^{2}}\right)^{2} \frac{\left|1-\Delta_{V}\right|^{2}+\left|r_{\mathrm{CP}}-\tilde{\Delta}_{V}\right|^{2}}{1+\left|r_{\mathrm{CP}}\right|^{2}}
$$

The parameters $\Delta_{V}$ and $\tilde{\Delta}_{V}$ contain the direct contributions and the corrections to the indirect contributions due to the off-shellness of the photon and the additional diagram with the Z-boson:

$$
\stackrel{(\sim)}{\Delta}_{V}=\stackrel{(\sim)}{\kappa}_{V} F_{\text {direct }}^{V}+\text { indirect corrections . }
$$

The direct form factors $F_{\text {direct }}^{V}$ can written in a similar form to the ones in eq. (4). The parameter $r_{\mathrm{CP}}$ vanishes in the $\mathrm{SM}$ and contains the various $\mathrm{CP}$-odd couplings to the indirect amplitude and the $h \rightarrow \gamma \gamma$ rate. The detailed expressions for both $F_{V}$ and $r_{\mathrm{CP}}$ can be found in ref. [2].

Table 2 quotes our SM branching ratios. Note that in the case of $h \rightarrow \Upsilon(1 S) \gamma$ the direct and indirect contributions cancel almost exactly, suppressing this decay by three orders of magnitude. This cancellation leads to a spectacular sensitivity of this branching ratio on $\kappa_{b}$ and $\tilde{\kappa}_{b}$, as demonstrated in 
Table 2. Predicted branching fractions for various $h \rightarrow V \gamma$ decays, including error estimates due to the uncertainties in the meson decay constants (" $f$ "), the direct contribution ("direct") and the branching ratio

$$
\operatorname{Br}(h \rightarrow \gamma \gamma) \text {. }
$$

\begin{tabular}{|c|c|}
\hline Decay mode & Branching ratio \\
\hline$h \rightarrow \rho^{0} \gamma$ & $\left(1.68 \pm 0.02_{f} \pm 0.08_{h \rightarrow \gamma \gamma}\right) \cdot 10^{-5}$ \\
$h \rightarrow \omega \gamma$ & $\left(1.48 \pm 0.03_{f} \pm 0.07_{h \rightarrow \gamma \gamma}\right) \cdot 10^{-6}$ \\
$h \rightarrow \phi \gamma$ & $\left(2.31 \pm 0.03_{f} \pm 0.11_{h \rightarrow \gamma \gamma}\right) \cdot 10^{-6}$ \\
$h \rightarrow J / \psi \gamma$ & $\left(2.95 \pm 0.07_{f} \pm 0.06_{\text {direct }} \pm 0.14_{h \rightarrow \gamma \gamma}\right) \cdot 10^{-6}$ \\
$h \rightarrow \Upsilon(1 S) \gamma$ & $\left(4.61 \pm 0.06_{f}^{+1.75}\right.$ direct $\left.\pm 0.22_{h \rightarrow \gamma \gamma}\right) \cdot 10^{-9}$ \\
$h \rightarrow \Upsilon(2 S) \gamma$ & $\left(2.34 \pm 0.04_{f}^{+0.75}\right.$ direct $\left.\pm 0.11_{h \rightarrow \gamma \gamma}\right) \cdot 10^{-9}$ \\
$h \rightarrow \Upsilon(3 S) \gamma$ & $\left(2.13 \pm 0.04_{f}^{+0.75}\right.$ direct $\left.\pm 0.10_{h \rightarrow \gamma \gamma}\right) \cdot 10^{-9}$ \\
\hline
\end{tabular}

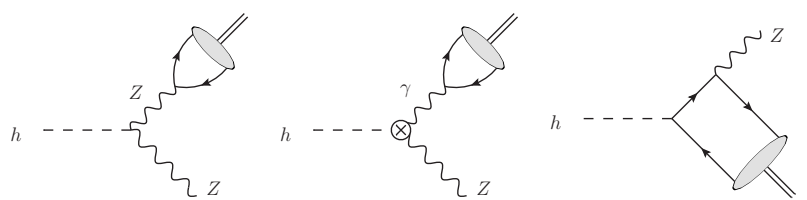

Figure 3. Diagrams contributing to the $h \rightarrow Z M$ decays. The suppression from the loops in the second diagram is lifted by the near on-shell photon.

figure 3 for the cases of $\tilde{\kappa}_{b}$ vanishing (left diagram) and not vanishing (right diagram). When compared to the branching ratio of $h \rightarrow \bar{b} b$, even information on the CP-phase of the Yukawa coupling of the $b$-quark can be obtained.

\subsection{Weak radiative Higgs decays}

Weak Higgs decays of the form $h \rightarrow V Z$ can be used to probe the coupling of the Higgs to a photon and a $Z$-boson, both loop-induced and from the couplings $\kappa_{\gamma Z}$ and $\tilde{\kappa}_{\gamma Z}$. There are three important contributions, shown in figure 3 . While naive dimensional analysis suggests that the tree-level diagram involving the $h Z Z$ gauge coupling is the dominant amplitude, the diagram with the loop-induced $h \gamma Z$ vertex is equally important. This is because the loop-suppression is overcome by the photon propagator being almost on its pole. For pseudoscalar mesons, the photon diagram does not exist and thus the decays $h \rightarrow P Z$ can only serve as a SM reference values.

The current limits on the parameters $\kappa_{\gamma Z}$ and $\tilde{\kappa}_{\gamma Z}$ from CMS [23] and ATLAS [24] imply upper bounds on the decay rates of 9 and 11 times the SM value, respectively, both at $95 \%$ confidence level. The stronger bound from CMS implies the constraint (under the assumption that all other parameters are at their SM value)

$$
\sqrt{\left|\kappa_{\gamma Z}-2.395\right|^{2}+\left|\tilde{\kappa}_{\gamma Z}\right|^{2}}<7.2 \text {. }
$$

Table 3 quotes our branching ratios both for the SM along with the maximal deviation when the bounds (9) are saturated. Of course it should be noted, that NP effects in $\kappa_{\gamma Z}$ and $\tilde{\kappa}_{\gamma Z}$ will also show in the direct $h \rightarrow \gamma Z$ measurement. 
Table 3. Predicted branching fractions for various $h \rightarrow V \gamma$ decays, including error estimates due to the uncertainties in the meson decay constants (" $f$ "), the direct contribution ("direct") and the branching ratio

$$
\operatorname{Br}(h \rightarrow \gamma \gamma) \text {. }
$$

\begin{tabular}{|c|c|c|}
\hline Decay mode & Branching ratio $\left[10^{-6}\right]$ & NP effects from $\kappa_{\gamma Z}$ \\
\hline$h \rightarrow \pi^{0} Z$ & $2.30 \pm 0.01_{f} \pm 0.09_{\Gamma}$ & - \\
$h \rightarrow \eta Z$ & $0.83 \pm 0.08_{f} \pm 0.03_{\Gamma}$ & - \\
$h \rightarrow \eta^{\prime} Z$ & $1.24 \pm 0.12_{f} \pm 0.05_{\Gamma}$ & - \\
$h \rightarrow \rho^{0} Z$ & $7.19 \pm 0.09_{f} \pm 0.28_{\Gamma}$ & $1.83-53.3$ \\
$h \rightarrow \omega Z$ & $0.56 \pm 0.01_{f} \pm 0.02_{\Gamma}$ & $0.06-4.56$ \\
$h \rightarrow \phi Z$ & $2.42 \pm 0.05_{f} \pm 0.09_{\Gamma}$ & $1.77-9.12$ \\
$h \rightarrow J / \Psi Z$ & $2.30 \pm 0.06_{f} \pm 0.09_{\Gamma}$ & $1.59-13.10$ \\
$h \rightarrow \Upsilon(1 S) Z$ & $15.38 \pm 0.21_{f} \pm 0.60_{\Gamma}$ & $13.7-20.8$ \\
\hline
\end{tabular}

\section{Conclusions}

Exclusive hadronic decays of heavy electroweak bosons are a theoretically clean playground for the framework of QCD factorization. The hard scale $\mu_{\text {hard }}$ is set by the decaying bosons and thus very high, rendering the impact of power corrections of $O\left(\Lambda_{\mathrm{QCD}} / \mu_{\text {hard }}\right)$ tiny. Additionally, the hadronic uncertainties are comparably small thanks to the renormalization group evolution of the LCDA. The price to pay is the smallness of the branching ratios. For the $Z$-decays, the most promising experimental environment would be a future lepton collider running at $\sqrt{s}=m_{Z}^{2}$, whereas the Higgs decays are an interesting physics target for the high-luminosity run of the LHC or a future $100 \mathrm{TeV}$ proton collider.

This report skips over a large amount of technical and phenomenological details, like a detailed derivation of the factorization theorem, the renormalization group evolution of the hadronic parameters and the special case of flavor-singlet mesons as well as the $W^{ \pm} \rightarrow M^{ \pm} \gamma$ and $h \rightarrow M^{ \pm} W^{\mp}$ decays [1-3]. We nevertheless hope to convince the reader that the physics opportunities in a dedicated program for the decays considered here are compelling, both within and beyond the standard model.

\section{Acknowledgements}

M.K is supported by the DFG Graduate School Symmetry Breaking in Fundamental Interactions (GRK 1581) and wants to thank the organizers of the workshop for the hospitality and a very interesting conference.

\section{References}

[1] Y. Grossman, M. König and M. Neubert, JHEP 1504 (2015) 101.

[2] M. König and M. Neubert, JHEP 1508 (2015) 012.

[3] S. Alte, M. König and M. Neubert, JHEP 1602 (2016) 162.

[4] S. Alte, M. König and M. Neubert, in preparation.

[5] G. Aad et al. [ATLAS Collaboration], Phys. Lett. B 716 (2012) 1.

[6] S. Chatrchyan et al. [CMS Collaboration], Phys. Lett. B 716 (2012) 30. 
[7] G. T. Bodwin, F. Petriello, S. Stoynev and M. Velasco, Phys. Rev. D 88 (2013) no.5, 053003.

[8] A. L. Kagan, G. Perez, F. Petriello, Y. Soreq, S. Stoynev and J. Zupan, Phys. Rev. Lett. 114 (2015) 10, 101802.

[9] G. P. Lepage and S. J. Brodsky, Phys. Lett. B 87 (1979) 359.

[10] G. P. Lepage and S. J. Brodsky, Phys. Rev. D 22 (1980) 2157.

[11] A. V. Efremov and A. V. Radyushkin, Theor. Math. Phys. 42 (1980) 97 [Teor. Mat. Fiz. 42,147 (1980)].

[12] A. V. Efremov and A. V. Radyushkin, Phys. Lett. B 94 (1980) 245.

[13] V. L. Chernyak and A. R. Zhitnitsky, Phys. Rept. 112 (1984) 173.

[14] C. W. Bauer, S. Fleming, D. Pirjol and I. W. Stewart, Phys. Rev. D 63 (2001) 114020.

[15] C. W. Bauer, D. Pirjol and I. W. Stewart, Phys. Rev. D 65 (2002) 054022.

[16] C. W. Bauer, S. Fleming, D. Pirjol, I. Z. Rothstein and I. W. Stewart, Phys. Rev. D 66 (2002) 014017.

[17] M. Beneke, A. P. Chapovsky, M. Diehl and T. Feldmann, Nucl. Phys. B 643 (2002) 431.

[18] M. V. Terentev, Sov. J. Nucl. Phys. 33 (1981) 911 [Yad. Fiz. 33, 1692 (1981)].

[19] T. Ohrndorf, Nucl. Phys. B 186 (1981) 153.

[20] M. A. Shifman and M. I. Vysotsky, Nucl. Phys. B 186 (1981) 475.

[21] V. N. Baier and A. G. Grozin, Nucl. Phys. B 192 (1981) 476.

[22] L. Bergstrom and G. Hulth, Nucl. Phys. B 259 (1985) 137 Erratum: [Nucl. Phys. B 276 (1986) 744].

[23] S. Chatrchyan et al. [CMS Collaboration], Phys. Lett. B 726 (2013) 587.

[24] G. Aad et al. [ATLAS Collaboration], Phys. Lett. B 732 (2014) 8. 